\title{
Analysis of Spatial and Temporal Evolution of Population Education Level in Lanzhou
}

\author{
Jing SHI ${ }^{1, a}$, Zhi-bin ZHANG ${ }^{1, *}$, Qian-qian GUO $^{1}$, Long CHEN $^{1}$, Ya-li ZHANG ${ }^{1}$ \\ ${ }^{1}$ Northwest Normal University, \\ Lanzhou, Gansu, China \\ a 519047808@qq.com
}

\begin{abstract}
Based on the way of mathematical statistics technology, Global spatial autocorrelation and center of gravity analysis, this paper choosing the 50 streets as research unit analyzed distribution dynamic evolution of the average education level by using the data of Lanzhou city census from 1982 to 2010. The results showed the following several aspects: (1)The proportion of the population in different education stages varied greatly from 1982 to 2010 ; (2)The average length of schooling has risen significantly. Among them, the education level of the population grew fastest between 1982 and 1990 and increased the most obviously from 2000 to 2010 . (3)The spatial structure was gradually transitioned from "Double-Center" to "Multi-Center", (4)The positive correlation of the streets was gradually weakened and the distribution of average education level tended to be balanced; (5) The center of gravity of population average educational level was moved to the southeast.
\end{abstract}

Keywords-the average number of years of schooling; space structure; spatial and temporal evolution; Lanzhou

\section{INTRODUCTION}

With the rapid development of social economy, people's living standards have improved significantly. The traditional manufacturing economy can no longer meet the needs of people. The knowledge economy is in urgent need of further expansion, which began to replace the traditional manufacturing economy gradually. As a special economic resource, power capital has been attached importance to by all walks of life in society, that is, the increase in quantity and the improvement in quality [1]. Population quality is the comprehensive performance of physical quality, cultural quality and ideological quality, in which cultural quality has gradually become a decisive factor affecting the comprehensive competitiveness of a region, and education is an important means to improve cultural quality. Education plays an extremely important role in the transformation of socio-economic development ${ }^{[2]}$. Since 1978, the educational cause of Lanzhou has developed rapidly, the cultural quality of the population has been greatly improved, and the overall popularization of compulsory education has been realized successively. The basic popularization of high school education and higher education enter the stage of popularization development ${ }^{[3]}$. According to the data of the sixth Census of Lanzhou in 2010, there are 657000 people with college education in the main urban area of Lanzhou. On average, the number of college students per million people rose from 67 in 1982 to 1103 in 2010, which shows that Lanzhou has achieved positive results in vigorously developing higher education, reflecting from the side the improvement in the level of education of the population. The average length of education can be used as indicators to reflect the education level of the population more comprehensively.

Using the continuity of education to estimate the education level, this method can put forward more reasonable assumptions for the distribution of educational system at each educational level, thus obtaining more accurate estimates of educational achievements and educational inequality ${ }^{[4]}$. In this paper, the main urban area of Lanzhou, which has a large population in the northwest, is taken as the research area, and the index system to measure the education level of the population is selected, which are analyzed comprehensively in terms of spatial distribution and internal structure changes of Lanzhou population from different levels, so as to analyze the evolution of urban internal social spatial structure. In order to balance regional development and talent planning and other related areas to provide a reference basis.

\section{Survey AND Data Processing OF the StUdy AREA}

\section{A. Survey of Research Area}

Lanzhou is located in the central part of Gansu Province. It is the center of politics, economy, culture, trade and trade in Gansu Province. It is also one of the most important central cities in Northwest China. In this paper, 49 streets and 1 high-tech development zone in Lanzhou Yellow River valley basin are selected as the study area, with a total area of $213.2 \mathrm{~km} 2$, which is located in the main urban area of Lanzhou (Chengguan, Anning, Xigu and Qilihe district located in the Yellow River valley basin of Lanzhou) ${ }^{[5]}$.

Because of the small interannual variation, this paper selects 1982, 1990, 2000 and 2010 as the main time nodes when studying the changes in the time series. Taking the average number of years of education of the population over the age of 6 as the main indicator, and the street as the minimum research unit, this paper analyzes the evolution of education level of Lanzhou population in recent 30 years. In this article, "Lanzhou" refers to the four districts of the main city; "streets" refer to the sub-district offices and the administrative area units at the township (town) level ${ }^{[6]}$.

\section{B. Data Processing}

Because the index of education level is not completely uniform in the data of the censuses, it is necessary to classify 
and process the education level of the population aged 6 years and over in the data. Considering a large number of data attributes and in order to better reflect the level of education of the population, it was decided to use the average number of years of schooling of the population as the only indicator, with the current number of years of schooling as the coefficient. The formula is as follows: average number of years of education $=$ proportion of population with university education degree $\times 16$ years + proportion of population with high school education degree $\times 12$ years + proportion of population with junior high school education degree $\times 9$ years + proportion of population with primary education degree $\times 6$ years + proportion of illiterate population $\times 0$ year (proportion of the population with various education levels is the proportion of population with this educational level to the total population) ${ }^{[7]}$.

\section{RESEARCH METHOD}

\section{A. Global Spatial Autocorrelation}

The spatial distribution of geographical things is always related to a certain extent ${ }^{[8]}$. The Moran's I index can be used to measure the spatial relationship between adjacent units, which reveal the spatial aggregation and dispersion characteristics of geographical units. The specific calculation formula is as follows:

$$
I=N \bullet \frac{\sum_{i} \sum_{j} W_{i j}\left(x_{i}-\bar{x}\right)\left(x_{j}-\bar{x}\right)}{\left(\sum_{i} \sum_{j} W_{i j}\right)\left(\sum_{i}\left(x_{i}-\bar{x}\right)^{2}\right.}
$$

In the form, $I$ is the global Moran's $I$ index, $N$ is the number of units in the study area, $W$ is the space weight, $x_{i}$ and $x_{j}$ are attributes of regions $i$ and $j, \quad \bar{x}$ is the average value of the attribute. If Moran's $I=0$, the space presents randomness. If Moran's $I>0$ and the closer it is to 1 , it means that its space is positively correlated, and the greater the value, the more obvious the spatial correlation is, and vice versa.

\section{B. Mean Pointer Center}

The center of population gravity refers to the point where the moment of the population distribution in the spatial plane reaches the balance at a certain time in the study area. By comparing with the center of gravity of the regional set, it is often used to measure the equilibrium of the population distribution in the region ${ }^{[2]}$. The formula is as follows:

$$
\bar{X}=\frac{\sum_{i}^{n} m_{i} x_{i}}{\sum_{i}^{n} m_{i}} \quad \bar{Y}=\frac{\sum_{i}^{n} m_{i} y_{i}}{\sum_{i}^{n} m_{i}}
$$

$x_{i}$ represents the latitude coordinates of $i$ street, $y_{i}$ represents the longitude coordinates of $i$ Street, $m_{i}$ represents the weight of ground $i, \bar{x}$ represents the latitude coordinates of the center of gravity, $\bar{x}$ represents the longitude coordinates of the center of gravity.

\section{Analysis on EVOlution of AVERAGE EduCATION LEVEL}

\section{A. Average Number of Years of Schooling Increased Rapidly}

The average number of years of education in Lanzhou continues to climb. In 1982, the average number of years of education in Lanzhou was 8.18. The average number of years of schooling for the population of Lanzhou in 1990 was 9.11, and between 1982 and 1990, the average number of years of schooling of the population increased by 0.93 year, and the ratio increased (relative to 1982) by $11.42 \%$, which was the highest increases in the population's level of education. The average number of years of schooling in Lanzhou was 10.11 in 2000 , an increase of about 1 year compared with the 1990 average, a year-on-year increase (relative to 1982) of $23.57 \%$, and a quarter increase (relative to 1990) reached 10.91\%. Compared with the previous phase (1982-1990), the average number of years of schooling increased from 1990 to 2000, while the growth rate slowed. In 2010, the population of Lanzhou had an education level of 11.23 years, the average number of years increased by 1.12 year, the annual increase (relative to 1982) reached $37.26 \%$, the ring increase (relative to 2000) was 11.08 , and the indicators increased significantly from the previous stage (2000-2010). This stage has become the most obvious one in which the average number of years of schooling has increased, with the extreme value of street differences reaching 5.56 years.

\section{B. "Dual-center" transition to "Multi-center"}

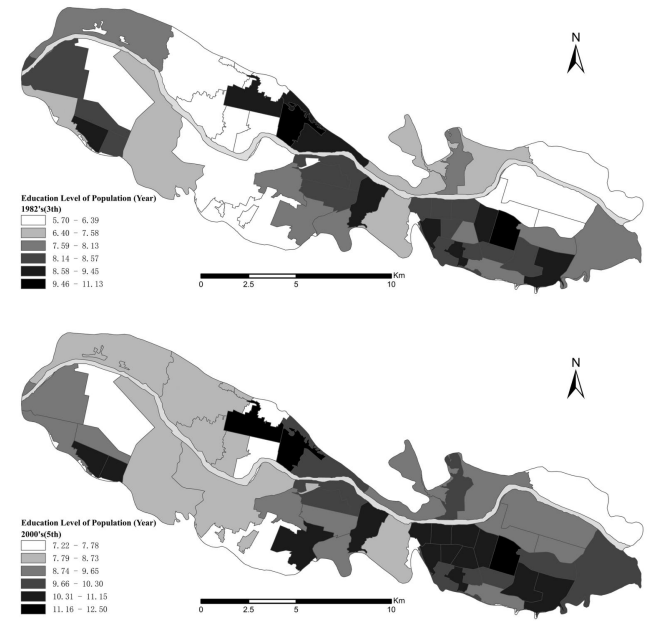

Fig. 1. Spatial distribution map of the average educational level of the population in Lanzhou 
As is shown in Fig.1, the average education level of Chengguan and Anning district is higher than that of the whole city, while that of Qilihe and Xigu district is lower than the average level of the whole city, which generally forms the spatial pattern of "two high and two low". In 1982, Chengguan district took Weiyuan Road and Railway West Village as the peak "center", Anning district Xilu, Peili and Shilidian Street as the peak "center", covering areas that coincided with the height of the streets where colleges are located. And the area with the obvious situation of surrounding high value together formed the city pass, the block high value area of tranquility small scope, present "double center" structure. In 1990, taking Weiyuan Road Street and Jiaojiawan Street in Chengguan district, Xiluand Peili Street in Anning district as its peak "center", the high-value areas of Chengguan district and Anning district were formed respectively. In 2000, the high-value area of Anning district remained unchanged, the high-value area of Chengguan district took Weiyuan road as the center, the scope gradually expanded, or the two high-value areas of Chengguan district and Anning district were dominant. By 2010, a gathering group consisting of Weiyuan Road Street in Chengguan district and its surrounding streets, Peili Street, Xilu Street, Yintan Road Street and Liujiabu Street in Anning district were formed into two high-value areas in the east and west, respectively. The Xilu Street and Gongjiawan Street, located in the central area of the city, are also high-value streets, showing a "multicenter" spatial structure as a whole.

By comparing the four spatial distribution maps of the average level of education, it can be found that most of the high peak values are located in the streets where colleges are concentrated, and the whole space presents the characteristics of co-existence of "more concentrated state" and "centrifugal diffusion". The high-level streets gradually spread from the center to the periphery, and gradually expanded from piecemeal blocks to flakes under the trend of increasing the coverage of high-level streets.

\section{Spatial Association Equalization}

The global Moran's I index reflects the distribution characteristics of the education degree of the population in the whole regional space, and the significance level of the Moran's $I$ index is tested by $Z$ value. In the data of four periods, the global Moran's $I$ value and $Z$ test value are both positive, and the $Z$ value is more than 1.96 , the $P$ value is less than 0.05 , and the confidence level is above 95\%. From 1982 to 2010, the distribution of the average educational level of Lanzhou population showed a positive spatial correlation, that is, in the streets with higher educational level, the population in the surrounding areas also had a higher level of education, and vice versa. The average educational level Moran's I value of Lanzhou population decreased from 0.1661 in 1982 to 0.107 in 2010 , indicating that with the increase of spatial distance, the positive correlation of spatial distribution of the average educational level gradually weakened. The spatial pattern of population education level changes from agglomeration type to equilibrium type.

\section{Center of Gravity Migration}



Fig. 2. The trajectory of the Center about Gravity of educated population in Lanzhou

As is shown in Fig.2, the results show that from 1982 to 2010 , the center of gravity of illiterate population gradually moved eastward, from the Qilihe Stadium of Jianlan Road Street in Qilihe district to the West Street of Xiaoxihu Lake, near the police station of Xiaoxihu Police Station, the center of gravity of the illiterate population moved eastward gradually. The center of gravity of the primary school population gradually moved eastward, and the migration span was relatively large, from the LANZHOU SHOPPING MALL to the nearby hospital of Lanzhou military region. The center of gravity of the junior middle school population shifted from the disease control center of Qilihe district to the northwest to LANZHOU SHOPPING MALL. After 1990, the center of gravity shifted to the southeast direction, and the migration span was large, all the way to the vicinity of municipal companies' home homes. The center of gravity migration of the high school population is also relatively large; from the power Electric Power Company to the Xihu primary school near, the migration process of the center of gravity to the east is faster and faster. The distance of the center of gravity of the university population is small, but the trajectory is relatively large. From 1982 to 2000 , the center of gravity of government family home of the Qilihe district moved to the southeast, reaching the vicinity of West Lake Park, and in 2010 it transferred to the northwest direction to reach the vicinity of the Xiaoxihu Yellow River Bridge. The whole move northward.

From 1982 to 2010, the center of gravity of Lanzhou population's education level did not change much, which was approximately located near West Lake Street. The whole evolution showed a relatively stable trend of concentric agglomeration, forming a moving track from north to south and slightly eastward at the same time.

\section{CONCLUSIONS}

In this paper, through the calculation and analysis of data and spatial, the following conclusions are drawn:

1. With regard to the number of years of schooling: The population has continued to rise in education, with a higher level of education in the Peili street of Anning district, Xilu and Weiyuan Road Street of Chengguan district, where the university is located; with an average lower level of education in the Qingbaishi and the Fulongping street of Chengguan district, the town of Pengjiaping and the Sijiqing street of Qilihe district. 
2. Spatial evolution: The pattern of the education level of population in Lanzhou is basically stable, and the spatial pattern of "two high and two low" is presented in the zoning. The gravity of the average educated population is gradually transitioned from "Double-center" to "Multi-center".

3. Spatial linkages and changes in the center of gravity: the positive correlation between the average level of education in the streets of Lanzhou is gradually weakened, the range of high-level streets is gradually expanding, the trend of agglomeration is gradually decreasing, and the spatial pattern of population education level is changed from agglomeration type to equilibrium type.

\section{REFERENCES}

[1] Chang-jun YUE. International Comparison of higher education and economic development in the 40 of reform and opening [J].Education and Economy, 2018(06):9-17. (In Chinese)

[2] Shan-yu ZHANG. Introduction to Population Geography [M].Shanghai: East China Normal University Press, 2013.2:283-284. (In Chinese)

[3] Shuang ZHANG. An Empirical Study on Education Level of Chinese Labor Force-Time Series Analysis Based on Census Data Platform [J]. Northwest population, 2014, 35(03): 73-78. (In Chinese)

[4] Vanesa Jordá, José M. Alonso. New Estimates on Educational Attainment Using a Continuous Approach (1970-2010) [J]. World Development, 2016.

[5] Zhi-bin ZHANG. Evolution Pattern and Regulation Path of Lanzhou Urban Population Spatial Structure[J]. Geographical Research, 2012, 31(11):2055-2068. (In Chinese)

[6] Lu MA, Spatial Distribution and Formation Mechanism of Aging Population in Lanzhou [J]. Human geography, 2015, 30 (06):132-137. (In Chinese)

[7] Fang WANG. Spatial model of population education in China and its timing evolution [D]. East China Normal University, 2012. (In Chinese)

[8] Lin Mei. Characteristics and causes of spatial and temporal differentiation of population aging in Jilin Province [J]. Progress in Geographical Science, 2018, 37(03):352-362.(In Chinese) 